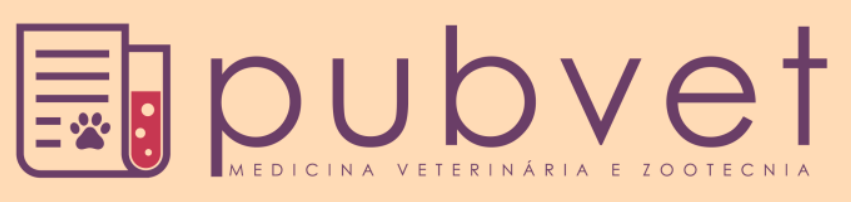

ISSN 1982-1263

https://doi.org/10.22256/pubvet.v12n6a115.1-5

\title{
Efetividade da educação sanitária na redução dos riscos no comércio de produtos cárneos
}

\author{
Cleber Bastos Rocha ${ }^{*} \bullet$, Antônio César Côrrea $^{2}{ }^{\bullet}$, Vivian Antunes Beneri ${ }^{3} \bullet$, Marília $^{\bullet}$ \\ da Costa Alvarengo ${ }^{4} \theta$, Fabio Moraes Miranda ${ }^{5} \bullet$, Michele Neves Meneses ${ }^{6} \theta$
}

\footnotetext{
${ }^{I}$ Médico Veterinário, Doutor em Ciências, Prefeitura Municipal de Rio Grande, Vigilância Sanitária, Rio Grande-RS, Brasil.

${ }^{2}$ Gestor Ambiental, Especialista em Gestão da Vigilância Sanitária, Fiscal Sanitário, Prefeitura Municipal de Rio Grande, Vigilância Sanitária, Rio Grande-RS, Brasil. E-mail:antonio.correa@riogrande.rs.gov.br

${ }^{3}$ Bacharel em Direito, Especialista em Gestão da Vigilância Sanitária, Fiscal Sanitário, Prefeitura Municipal de Rio Grande, Vigilância Sanitária, Rio Grande-RS, Brasil.E-mail: viviansms@ riogrande.rs.gov.br

${ }^{4}$ Bacharel em Biologia, Especialista em Gestão da Vigilância Sanitária. E-mail: marilia.alvarengo@gmail.com

${ }^{5}$ Fiscal Sanitário, Prefeitura Municipal de Rio Grande, Vigilância Sanitária, Rio Grande-RS, Brasil. E-mail: fabio.miranda@ riogrande.rs.gov.br

${ }^{6}$ Bacharel em Enfermagem, Especialista em Vigilância em Saúde Ambiental, Prefeitura Municipal de Rio Grande, Centro de Vigilância em Saúde, Rio Grande-RS, Brasil. E-mail: michele.sms@ riogrande.rs.gov.br

*Autor para correspondência, E-mail: clebersms@riogrande.rs.gov.br
}

RESUMO. O consumo de produtos cárneos sem procedência ou sem condições de boas práticas de manipulação põem em risco a saúde população. Desta forma, a educação pode ser uma ferramenta importante para melhoria das condições sanitárias do comércio de produtos cárneos. O objetivo deste trabalho foi avaliar a eficiência de uma ação educativa na melhoria da qualidade dos produtos oferecidos em açougues. Foi disponibilizada, via formulário eletrônico, uma pesquisa de opinião ao público, sobre a qualidade dos produtos cárneos encontrados no comércio local. Também foi realizada uma palestra educativa para 23 estabelecimentos (manipuladores e proprietários), com uma avaliação de conhecimentos pré e pós-palestra. Os estabelecimentos também foram classificados em Ruim, Regular ou Bom, através de inspeção sanitária in loco por meio de lista de verificação. A pesquisa de opinião demonstrou a preocupação da população quantos aos riscos sanitários no consumo carnes. A avaliação dos manipuladores/proprietários revelou um bom conhecimento prévio, com aprimoramento no tema após a palestra. Verificou-se através das inspeções, uma melhora das condições sanitárias após a atividade educativa. Conclui-se que, a educação sanitária contribui para redução dos riscos no comércio de produtos cárneos.

Palavras-chave: açougues, carnes, educação, sanitária, vigilância

\section{Health education of food handlers in the meat products market}

ABSTRACT. The consumption of meat products without a reliable provenance or good handling practices may result in high risk of diseases to the population. Thus, the health education can be a useful tool for the improvement of sanitary conditions of the meat products market. This study is intended to evaluate the efficiency of an educational activity towards the improvement of the quality of products offered in butchery. A public opinion survey regarding the quality of meat products in local marketplaces has been provided via Electronic Form. Moreover, an educational lecture for 24 establishments (food handlers and owners) has been given, with a pre- and post-knowledge evaluation. The establishments were classified as Bad, Regular or Good via in loco sanitary inspection through checklist. The public survey exposed the concerns of the population regarding the sanitary risks in meat products consumption. The food handlers/owners evaluation indicated a good previous knowledge, along with enrichment in the topic after 
the lecture. Through the inspections, an improvement in sanitary conditions has been verified after the educational activity. In conclusion, health education contributes to the reduction of risks in the meat product market.

Keywords: butchery, meat, education, health, surveillance

\section{Eficacia de la educación sanitaria en la reducción de los riesgos en el comercio de productos cárnicos}

RESUMEN. El consumo de productos cárnicos sin procedencia o sin condiciones para buenas prácticas para manipulación pone en riesgo la salud de la población. De esta forma, la educación puede ser una herramienta importante para mejorar las condiciones sanitarias del comercio de productos cárnicos. El objetivo de este trabajo es evaluar la eficiencia de una acción educativa en la mejora de la calidad de los productos ofrecidos en carnicerías. Se proporcionó, a través del formulario electrónico, una encuesta de opinión al público, sobre la calidad de los productos cárnicos encontrados en el comercio local. También se realizó una conferencia educativa para 23 establecimientos (manipuladores y propietarios), con una evaluación de conocimientos pre y postconferencia. Los establecimientos también fueron clasificados en Ruim, Regular o Bueno, a través de inspección sanitaria in loco por medio de una lista de verificación. La encuesta de opinión demostró la preocupación de la población cuántos a los riesgos sanitarios en el consumo de carne. La evaluación de los manipuladores / propietarios reveló un buen conocimiento previo, con perfeccionamiento en el tema después de la conferencia. Se verificó a través de las inspecciones, una mejora de las condiciones sanitarias después de la actividad educativa. Se concluye que la educación sanitaria contribuye a reducir los riesgos en el comercio de productos cárnicos.

Palabras clave: carnicería, carnes, educación, sanitaria, vigilancia

\section{Introdução}

O desconhecimento dos riscos sanitários por parte dos manipuladores de alimentos pode ser um entrave para qualidade do produto oferecido ao consumidor, podendo gerar graves prejuízos a saúde pública. As doenças transmitidas por alimentos constituem um problema de saúde pública em nível mundial, e estão frequentemente associadas à falhas na manipulação dos alimentos (Devides et al., 2014)

A educação sanitária é uma das ferramentas que busca a prevenção e a garantia da segurança alimentar, e através dela pode-se obter a conscientização dos manipuladores na responsabilidade de suas ações.

A carne bovina em cortes e moída "in natura" tem sido reconhecida como fonte primária de infecção quando manipulada incorretamente, ocasionando graves consequências à saúde dos seres humanos, tanto para os próprios manipuladores como os consumidores (Almeida et al., 2010).

Segundo dados da Secretaria de Segurança pública do RS, de Janeiro a Junho de 2017 foram registrados 3.940 ocorrências de crime de abigeatos no Rio Grande do Sul, somente no município de Rio Grande foram 59 registros neste período (RS, 2017).

O consumo de produtos sem fiscalização como carnes e produtos lácteos deixam a população exposta a um elevado número de zoonoses entre elas complexo teníase/cisticercose, tuberculose, listeriose, brucelose, salmoneloses, dentre outras.

Além da transmissão de doenças, há risco de outros agravos relacionados a resíduos tóxicos, fármacos, anabolizantes e hormônios (Abrahao et al., 2005; Soares et al., 2010).

Desta forma, o objetivo deste trabalho foi avaliar a efetividade da educação na redução dos riscos sanitários no comércio de produtos cárneos do município de Rio Grande-RS.

\section{Material e métodos}

Com a população consumidora realizou-se uma pesquisa de opinião, durante 30 dias, divulgado através das redes sociais. Em cinco questionamentos buscou-se obter dados sobre a origem do participante, qualidade da carne 
adquirida pelo consumidor e a preferência na forma de comercializar. Por fim, questionamos se consideravam importante o treinamento dos açougueiros pela vigilância sanitária.

Quanto ao setor regulado, a pesquisa foi realizada em oito bairros localizados na área periférica da cidade de Rio Grande-RS (Parque Marinha, Parque São Pedro, Carreiros, Santa Rosa, Castelo Branco, Profilurb II, Aeroporto e Vila Maria), totalizando 24 estabelecimentos de comércio de carnes (açougues) e minimercados.

O trabalho teve início com a inspeção das condições estruturais e sanitárias, através de uma lista de verificação (Inspeção Sanitária Inicial). Composta por 17 itens, como presença de alvará sanitário, higiene, temperatura dos produtos, entre outras exigências encontradas no Decreto Estadual/RS n²3430/1974. Cada item teve uma pontuação pré-determinada, de acordo com o risco sanitário, variando de 10 até 30 pontos. Através desta pontuação se classificou os estabelecimentos como Ruim (0-229), Regular (230-270) e Bom (280-370).

Para realização das palestras os estabelecimentos foram divididos de forma homogênea quanto a pontuação em dois grupos (A e B), totalizando 12 estabelecimentos em cada dia. Foram convidados dois participantes por estabelecimento sendo um proprietário ou gerente e um manipulador. A participação do proprietário/gerente foi estimulada visto que muitas vezes o funcionário não tem poder de decisão, principalmente quando as melhorias nas atividades envolvem aspectos financeiros.

No dia da palestra os convidados responderam a uma Avaliação Inicial com 16 questões subdividida em dois temas: Manipulação, estrutura e conservação; Higiene pessoal, alimentar e doenças de origem alimentar. Além disso, houve atualização sobre a legislação no comércio de carnes e noções sobre crimes ao consumo. Ao término das palestras, foi aplicado o mesmo questionário inicial (Avaliação Final).

De acordo com número de acertos, os participantes foram classificados com nível de conhecimento dos temas em Ruim 0-4, Regular 5-8, Bom 9-12 e Excelente 13-16.

Passados 30 dias, foram realizadas novas vistorias nos estabelecimentos (Inspeção Sanitária Final) para observar se ocorreu mudanças, ou seja, melhorias em razão da atividade educativa.

Os dados foram analisados através do teste de $\mathrm{T}$ de Student, ao nível de 5\% de probabilidade para verificar se havia diferença significativa entre os eventos (antes $\mathrm{x}$ depois das palestras).

\section{Resultados e discussão}

A pesquisa de opinião teve a participação de 458 consumidores. Como resultados, 78,6\% dos participantes residiam no município de Rio Grande-RS sendo os demais de outros municípios. Entre estes, 50,4\% dos consumidores responderam já terem adquirido produtos cárneos estragados. Quanto à carne moída, 76,9\% prefere que seja processada na hora da compra, mesmo que tenha que esperar e $86 \%$ dos pesquisados pagariam mais por um produto de maior qualidade. E, quanto ao tema educação sanitária para açougueiros, 94,5\% acha importante o treinamento por parte da vigilância sanitária.

Desta forma, infere-se que o investimento em tempo de treinamento e qualidade dos produtos oferecidos pelo comércio, tem notória preferência por essa parcela da população, mesmo que demande maior custo ao consumidor. Resultados semelhantes foram observados por Velho et al. (2009), quando avaliaram se o consumidor pagaria mais por uma carne certificada.

$\mathrm{Na}$ Tabela 1 encontramos o resultado da Avaliação Inicial e Final dos testes aplicados aos ouvintes da palestra.

$\mathrm{Na}$ Avaliação Inicial somente $7,15 \%$ dos ouvintes teve nota regular, enquanto a maioria foi classificada como excelente, demonstrando grau de conhecimento prévio das normas sanitárias.

Tabela 1. Classificação do nível de conhecimento dos ouvintes da palestra em função da percentagem de respostas certas, antes e após a aplicação das palestras.

\begin{tabular}{lcccccccccc}
\hline Turmas & \multicolumn{4}{c}{ Avaliação Inicial $(\%)$} & \multicolumn{3}{c}{ Avaliação Final } \\
\hline & Ruim & Regular & Bom & Excelente & Total & Ruim & Regular & Bom & Excelente & Total \\
A & - & - & 29,42 & 70,58 & 100 & - & - & 11,77 & 88,23 & 100 \\
B & - & 7,15 & 35,71 & 57,14 & 100 & - & - & 28,51 & 71,42 & 100 \\
\hline
\end{tabular}

Classificação quantos aos acertos: Ruim 0-4; Regular 5-8; Bom 9-12; Excelente 13-16. Turma A n= 17, Turma B n=14. 
Mesmo assim, com relação à porcentagem média da Avaliação Final houve um aumento nos acertos. Isso porque, os temas abordados ocasionaram interesse para aquisição do conhecimento. Comparável ao estudo de Araújo et al. (2011), em que os manipuladores de alimentos apresentaram um resultado muito bom quanto à aquisição de novos conhecimentos.

$\mathrm{Na}$ Tabela 2 observamos as médias dos acertos na avaliação Inicial e Final de acordo com os temas, onde se observa diferença estatística $(\mathrm{P}<0,05)$ para ambas as turmas.

Nos dois temas propostos, a média entre a Avaliação Inicial e Final aumentou significativamente $(\mathrm{P}<0,05)$, ressaltando a importância das ações educativas. Em sua pesquisa sobre o complexo teniose-cisticercose e boas práticas de manipulação dos alimentos, Izola et al. (2014) verificaram que os profissionais de açougue desconheciam o assunto, sinalizando falta de capacitação para identificar possíveis perigos. O que corrobora com o exposto nesse trabalho.

Tabela 2. Média e desvio padrão de acertos na Avaliação Inicial e Final aplicados aos ouvintes nos dois temas propostos.

\begin{tabular}{llll}
\hline Turmas & \multicolumn{1}{c}{ Temas* } & \multicolumn{1}{c}{$\begin{array}{c}\text { Avaliação } \\
\text { Inicial }\end{array}$} & $\begin{array}{c}\text { Avaliação } \\
\text { Final }\end{array}$ \\
\hline A & $\begin{array}{l}\text { Manipulação, estrutura } \\
\text { conservação. }\end{array}$ & $\mathrm{e}_{6,35 \pm 1,05^{\mathrm{a}}}$ & $6,64 \pm 0,93^{\mathrm{b}}$ \\
$\mathrm{A}$ & $\begin{array}{l}\text { Higiene pessoal, alimentar } \\
\text { doenças de origem alimentar. }\end{array}$ & $\mathrm{e}_{7,35 \pm 0,70^{\mathrm{a}}}$ & $7,64 \pm 0,60^{\mathrm{b}}$ \\
$\mathrm{B}$ & $\begin{array}{l}\text { Manipulação, estrutura } \\
\text { conservação. }\end{array}$ & $\mathrm{e}_{6,07 \pm 1,43 \mathrm{a}} 6,64 \pm 1,44^{\mathrm{b}}$ \\
$\mathrm{B}$ & $\begin{array}{l}\text { Higiene pessoal, alimentar } \\
\text { doenças de origem alimentar. }\end{array}$ & $6,85 \pm 1,29^{\mathrm{a}}$ & $7,64 \pm 0,74^{\mathrm{b}}$ \\
\hline
\end{tabular}

*Total 16 questões, 8 por tema. Letras distintas entre as colunas demonstram diferença estatística através do $\mathrm{T}$ de Student, ao nível de 5\%. Turma A $\mathrm{n}=17$ participantes, Turma B $\mathrm{n}=14$ participantes.

Na Tabela 3 são apresentados dados obtidos com a pontuação resultante da inspeção sanitária pré e pós-palestra.

Os estabelecimentos tiveram melhora na pontuação das inspeções após a palestra $(\mathrm{P}<$ $0,05)$, evidenciando a importância da educação sanitária.

Mesmo com essa melhora, entre os 23 estabelecimentos inspecionados, foram lavrados nove autos de infração sanitária, resultando na apreensão de $338,95 \mathrm{~kg}$ de produtos cárneos. A principal infração encontrada foi o processo de industrialização de carnes, como o congelamento e produção de carnes salgadas (charque). Os comerciantes infratores alegaram que estes processos eram realizados para evitar perdas econômicas, visto a dificuldade, por exemplo, na venda de picanha e filé mignon, que eram reservados através de congelamento, para venda posterior.

Tabela 3. Classificação dos estabelecimentos quanto as condições de estrutura e higiênico-sanitária

\begin{tabular}{lcc}
\hline $\begin{array}{l}\text { Classificação do } \\
\text { estabelecimento }\end{array}$ & $\begin{array}{c}\text { Pré-palestra } \\
(\%)\end{array}$ & $\begin{array}{c}\text { Pós-palestra } \\
(\%)\end{array}$ \\
\hline Ruim (0-229) & $39,14 \mathrm{a}$ & $8,69 \mathrm{~b}$ \\
Regular (230-279) & $30,43^{\mathrm{a}}$ & $39,14 \mathrm{~b}$ \\
Bom (280-370) & $30,43^{\mathrm{a}}$ & $52,17 \mathrm{~b}$ \\
\hline
\end{tabular}

Letras distintas na mesma linha demonstram diferença estatística através do T de Student, ao nível de 5\%, n=23.

Também quanto ao congelamento, alguns alegavam que as carnes estragavam em pouco tempo, reservando recortes de desossa para produzir carne moída. Cabe salientar, que a má manipulação influencia a qualidade sanitária da matéria-prima. Em seu estudo, Damer et al. (2014) avaliaram a qualidade da carne moída em 14 estabelecimentos, observando que todas estavam contaminadas por coliformes totais e termotolerantes, indicando precárias condições de higiene no processamento. Quanto as carnes salgadas, como não havia justificativa, os mesmos alegavam desconhecimento da lei.

O congelamento ou salga de carnes desossadas no próprio estabelecimento, além de influenciar negativamente na qualidade do produto, dificultam a identificação da origem, podendo ser subterfúgio para inclusão de carnes clandestinas, sejam elas provindas de abigeatos ou abate sem inspeção. Mathias (2008), através do modelo de oferta e demanda estima uma clandestinidade do abate de bovinos no Brasil em torno de $40 \%$. Desta forma, a ação da vigilância neste setor se torna cada vez mais importante.

A partir dos dados obtidos, pôde-se observar que a maioria dos estabelecimentos se julga com certo grau de conhecimento em relação aos itens questionados, porém se percebe resistência na aplicação das normas em relação ao armazenamento e conservação destes produtos. 


\section{Conclusões}

As ações da vigilância sanitária em educação resultaram uma elevada melhoria nas condições higiênicossanitária dos estabelecimentos.

\section{Referências bibliográficas}

Abrahao, R. M. C. M., Nogueira, P. A. \& Malucelli, M. I. C. 2005. O comércio clandestino de carne e leite no Brasil e o risco de transmissão da tuberculose bovina e de outras doenças ao homem: um problema de saúde pública. Archives of Veterinary Science, 10, 1-17.

Almeida, A. C., Souza, R. M., Pinho, L., Sobrinho, E. M., Silva, B. C. M. 2010. Determinação de perigos microbiológicos em carnes bovinas resfriadas provenientes de abates clandestinos e abate ilegal. Acta Veterinaria Brasilica, 4(4), 278-285.

Araújo, W. D. B., Deus, A. E., Santos, C. E. M., Pizziolo, V. R. \& Almeida, M. E. F. 2011. Avaliação do conhecimento de manipuladores de alimentos antes e depois de palestras educativas. Revista Vivências, 7, 23-36.

Damer, J. R. S., Dill, R. E., Gusmão A. A \& Moresco T. R. 2014. Contaminação de Carne Bovina Moída por Escherichia coli e Salmonella sp. Revista Contexto e Saúde, 14, 20-27.

Devides, G. G. G., Maffei, D. F. \& Catanozi, M.P.L.M. 2014. Perfil socioeconômico e profissional de manipuladores de alimentos e o impacto positivo de um curso de capacitação em boas práticas de fabricação. Brazilian Journal of Food Technology, 17, 166-176.
Izola, B. F., Rossi, G. A. M., Picinato, M. A. C., Hoppe, E. G. L., Martins, A. M. C. V. \& Bürger, K. P. 2014. Conhecimento de manipuladores de carne sobre o complexo teniose-cisticercose e boas práticas de manipulação de alimentos em Jaboticabal, São Paulo, Brasil. Revista Brasileira de Ciência Veterinária, 21,127-130.

Mathias, J. F. C. M. A. 2008. Clandestinidade na produção de carne bovina no Brasil. Revista de Política Agrícola. Ano XVII, 1.

Rio Grande do Sul, Secretaria da Segurança Pública. 2017. Departamento de Planejamento e Políticas de Segurança Pública, Observatório Estadual da Segurança Pública. Disponível em: <http://www.ssp.rs.gov.br/indicadorescriminais>. Acesso em: 12 Nov. 2017.

Soares, K. M. P., Leite, A. I. \& Bezerra, N. M. 2010. Importância do Médico Veterinário no controle do complexo teníase-cisticercose. Publicações em Medicina Veterinária $e$ Zootecnia, 4, 746.

Velho, J. P., Barcellos, J. O. J., Lengler, L., Elias, S.A. \& Oliveira, T. E. 2009. Disposição dos consumidores porto-alegrenses à compra de carne bovina com certificação. Revista Brasileira de Zootecnia, 38, 399-404.

Article History:

Received 20 April 2018

Accepted 15 May 2018

Available online 31 May 2018

License information: This is an open-access article distributed under the terms of the Creative Commons Attribution License 4.0, which permits unrestricted use, distribution, and reproduction in any medium, provided the original work is properly cited. 